\title{
AUTOMORPHISMS OF THE HATCHER-THURSTON COMPLEX
}

\author{
ELMAS IRMAK AND MUSTAFA KORKMAZ
}

\begin{abstract}
Let $S$ be a compact, connected, orientable surface of positive genus. Let $\mathcal{H} \mathcal{T}(S)$ be the Hatcher-Thurston complex of $S$. We prove that $\operatorname{Aut} \mathcal{H} \mathcal{T}(S)$ is isomorphic to the extended mapping class group of $S$ modulo its center.
\end{abstract}

\section{INTRODUCTION}

Let $S$ be a compact, connected, orientable surface of genus $g$ with $r \geq 0$ boundary components. The extended mapping class group, $\operatorname{Mod}_{S}^{*}$, of $S$ is the group of isotopy classes of all homeomorphisms (including orientation reversing) of $S$. The group $\operatorname{Mod}_{S}^{*}$ can be viewed as the automorphism group of various geometric objects. These objects include the complex of curves, the complex of nonseparating curves, the complex of separating curves, the complex of pants decompositions and the complex of Torelli geometry.

The Hatcher-Thurston complex $\mathcal{H} \mathcal{T}(S)$, which is defined in Section 2 below, plays a special role in the theory of mapping class groups. This complex was constructed in $\mathrm{HT}$ ] by A. Hatcher and W. Thurston (and used by B. Wajnryb W1] in order to find a presentation for the mapping class group. It was also used by J. Harer $\mathrm{Ha}$ in his computation of the second homology group of mapping class group. There is a natural action of $\operatorname{Mod}_{S}^{*}$ on $\mathcal{H} \mathcal{T}(S)$ by automorphisms. The purpose of this paper is to show that every automorphism of $\mathcal{H} \mathcal{T}(S)$ is induced by some element of $\operatorname{Mod}_{S}^{*}$. More precisely, we prove that the automorphism group of the Hatcher-Thurston complex $\mathcal{H} \mathcal{T}(S)$ is isomorphic to the group $\operatorname{Mod}_{S}^{*}$ modulo its center. We do this by proving that the automorphism group of our complex is isomorphic to the automorphism group of the complex $\mathcal{G}(S)$ on nonseparating simple closed curves, the complex defined by P. Schmutz Schaller in Sc]. (See Section 2 for the definition of $\mathcal{G}(S)$.)

Date: November 6, 2018.

1991 Mathematics Subject Classification. Primary 57M99; Secondary 20F38.

Key words and phrases. Mapping class groups, Hatcher-Thurston complex, Complex of curves.

The first author is supported by a Rackham Faculty Fellowship, Horace H. Rackham School of Graduate Studies, University of Michigan. The second author is supported in part by the Turkish Academy of Sciences under the Young Scientists Award Program (MK/TÜBA-GEBİ 2003-10). 
Another complex, the so-called complex of curves $C(S)$, was introduced about the same time by W. Harvey $[\mathrm{H}]$. It was also proved to be of the fundamental importance in the topology of surfaces and in the theory of Teichmüller spaces. Its automorphisms were investigated in the pioneering paper of N. Ivanov $[\mathrm{I}$, who proved that the group of automorphisms of $C(S)$ is equal to the extended mapping class group of $S$ (for genus $>1$ ), and found important applications of this result to the mapping class groups and to the Teichmüller spaces. His result was used to find automorphisms groups of various other objects related to surfaces (see [BM], CC, [FI, [Ir3, [M], $\mathrm{MW}, \underline{\mathrm{Sc}}$ ) and inspired some generalizations (see [r1], Ir2], [K], L]). The paper of D. Margalit [M] deals with an object closest to the one considered by us, namely with the so-called pants complex. He proved that the automorphism group of the pants complex is isomorphic to the extended mapping class group. While the results of $[\mathrm{M}]$ and of this paper are similar in the spirit, neither of them implies the other.

Here is how we prove our main result, Theorem 10. The vertices of the Hatcher-Thurston complex $\mathcal{H} \mathcal{T}(S)$ are cut systems. We encode nonseparating simple closed curves by vertices and edges of $\mathcal{H} \mathcal{T}(S)$. A great deal of work is on this choice. Using this coding, we show that the automorphism group of $\mathcal{H} \mathcal{T}(S)$ has a well-defined action on the set of (isotopy classes of) nonseparating simple closed curves. Under this action, we show that dual circles are mapped to dual circles, giving rise to a homomorphism from the group of automorphisms of $\mathcal{H} \mathcal{T}(S)$ to that of the complex $\mathcal{G}(S)$. We prove that this homomorphism is in fact an isomorphism. We want to point out that our proof and the proof of the main result of $[\mathrm{M}]$ are independent of each other, but have some similarities. (The similarities are pointed out to us by Margalit.) The similarities are perhaps not surprising, as these methods are indeed very natural to use in this situation.

The paper is organized as follows. In Section 2 we give the definition of various complexes used in the paper and state the relevant properties of these complexes. In Section [3, we show that every automorphism $f$ of the Hatcher-Thurston complex $\mathcal{H} \mathcal{T}(S)$ induces an automorphism $\tilde{f}$ of the complex $\mathcal{G}(S)$. Finally, in Section 4 , we state and prove the main theorem, and discuss some alternative approach.

\section{VARIOUS COMPleXes ON CURVES}

A simple closed curve on $S$ is said to be nontrivial (or essential) if it does not bound a disk on $S$ and it is not homotopic to a boundary component of $S$. We denote simple closed curves by capital letters and their isotopy classes by the corresponding lowercase letters. The geometric intersection number $i(a, b)$ of two classes $a$ and $b$ is defined as the minimum number of intersection points of $A$ and $B$ for $A \in a$ and $B \in b$.

We denote by $\mathcal{A}$ the set of isotopy classes of nontrivial simple closed curves on $S$. If $C$ is a simple closed curve on $S$, the surface obtained from $S$ by 
cutting along $C$ is denoted by $S_{C}$. Any two simple closed curves $A, B$ are always assumed to intersect each other minimally. We say that two simple closed curves $A$ and $B$ on $S$ are dual if they intersect each other transversely at only one point. In this case we also say that their isotopy classes $a$ and $b$ are dual. We note that for a simple closed curve $A$ there is a curve dual to $A$ if and only if $A$ is nonseparating.

The Hatcher-Thurston complex. Let $C_{1}, C_{2}, \ldots, C_{g}$ be pairwise disjoint nonseparating simple closed curves on $S$ such that the surface obtained from $S$ by cutting along all $C_{i}$ is connected, so that it is a sphere with $2 g+r$ boundary components. We call the set $\left\{c_{1}, c_{2}, \ldots, c_{g}\right\}$ a cut system and denote it by $\left\langle c_{1}, c_{2}, \ldots, c_{g}\right\rangle$.

Let $v$ and $w$ be two cut systems. Suppose that there are $c \in v$ and $d \in w$ such that $i(c, d)=1$ and $v-\{c\}=w-\{d\}$. We say that $w$ is obtained from $v$ by an elementary move and we write $v \leftrightarrow w$.

If $\left\langle c_{1}, c_{2}, \ldots, c_{i}, \ldots, c_{g}\right\rangle \leftrightarrow\left\langle c_{1}, c_{2}, \ldots, c_{i}^{\prime}, \ldots, c_{g}\right\rangle$ is an elementary move, then we drop the unchanged curves from the notation and write $\left\langle c_{i}\right\rangle \leftrightarrow\left\langle c_{i}^{\prime}\right\rangle$.

Let $\mathcal{H} \mathcal{T}^{1}(S)$ be the graph obtained by taking cut systems on $S$ as the vertex set and pairs of vertices $\{v, w\}$ such that $v \leftrightarrow w$ as the (unordered) edges. This will be the 1-skeleton of the Hatcher-Thurston complex.

A sequence of cut systems $\left(v_{1}, \ldots, v_{n}\right)$ forms a path in $\mathcal{H} \mathcal{T}^{1}(S)$ if every consecutive pair in the sequence is connected by an edge in $\mathcal{H} \mathcal{T}^{1}(S)$. A path is closed if $v_{1}=v_{n}$. There are three types of distinguished closed paths in the graph $\mathcal{H} \mathcal{T}^{1}(S)$.

Triangles. If three vertices have $g-1$ common elements and if the remaining classes $c, c^{\prime}, c^{\prime \prime}$ satisfy $i\left(c, c^{\prime}\right)=i\left(c, c^{\prime \prime}\right)=i\left(c^{\prime}, c^{\prime \prime}\right)=1$, then

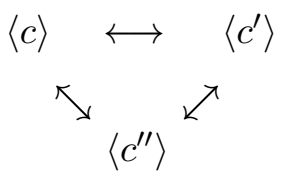

is a triangle (c.f. Figure 1 (i)). We denote this triangle by $\langle c\rangle \leftrightarrow\left\langle c^{\prime}\right\rangle \leftrightarrow$ $\left\langle c^{\prime \prime}\right\rangle \leftrightarrow\langle c\rangle$.

Rectangles. If four vertices have $g-2$ common elements and if the remaining classes $c_{1}, c_{2}, d_{1}, d_{2}$ have representatives $C_{1}, C_{2}, D_{1}, D_{2}$ as in Figure 1 (ii), then

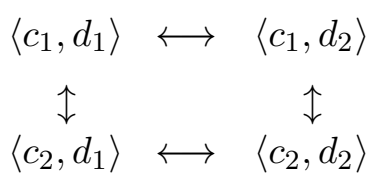

is a rectangle. We denote this rectangle by $\left\langle c_{1}, d_{1}\right\rangle \leftrightarrow\left\langle c_{1}, d_{2}\right\rangle \leftrightarrow\left\langle c_{2}, d_{2}\right\rangle \leftrightarrow$ $\left\langle c_{2}, d_{1}\right\rangle \leftrightarrow\left\langle c_{1}, d_{1}\right\rangle$. 
Pentagons. If five vertices have $g-2$ common elements and if the remaining classes $c_{1}, c_{2}, c_{3}, c_{4}, c_{5}$ have representatives $C_{1}, C_{2}, C_{3}, C_{4}, C_{5}$ intersecting each other as in Figure 1(iii), then

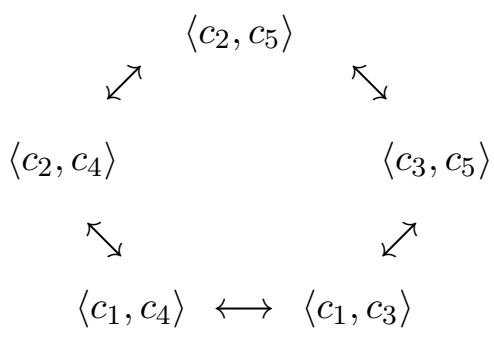

is a pentagon. Similar to triangles and rectangles we denote this pentagon by $\left\langle c_{1}, c_{4}\right\rangle \leftrightarrow\left\langle c_{2}, c_{4}\right\rangle \leftrightarrow\left\langle c_{2}, c_{5}\right\rangle \leftrightarrow\left\langle c_{3}, c_{5}\right\rangle \leftrightarrow\left\langle c_{1}, c_{3}\right\rangle \leftrightarrow\left\langle c_{1}, c_{4}\right\rangle$.

The Hatcher-Thurston complex $\mathcal{H} \mathcal{T}(S)$ is a two-dimensional CW-complex obtained from $\mathcal{H T}^{1}(S)$ by attaching a 2-cell along each triangle, rectangle and pentagon.

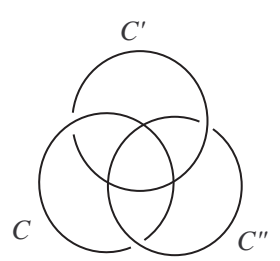

(i)

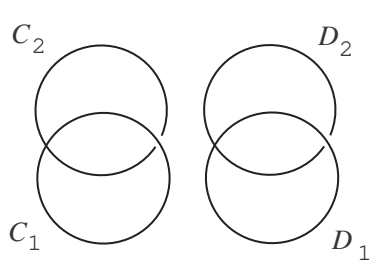

(ii )

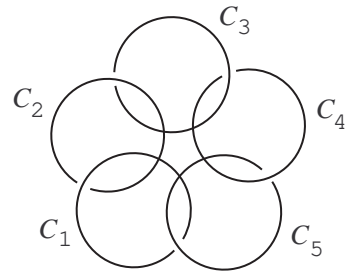

( iii )

Figure 1. A triangle, a rectangle and a pentagon in the Hatcher-Thurston complex

Hatcher and Thurston used this complex to get a presentation for the mapping class group for closed orientable surfaces, $[\mathrm{HT}$. They proved that $\mathcal{H} \mathcal{T}(S)$ is connected and simply connected. Wajnryb used it to get a simple presentation for the mapping class group W1 and he also gave an elementary proof of the connectivity and the simple connectivity of this complex in W2].

Theorem 1. ([HT, W2]) Let $S$ be a compact, connected, orientable surface of genus at least one. Then the complex $\mathcal{H} \mathcal{T}(S)$ is connected.

The complexes of curves. The complex of curves, $\mathcal{C}(S)$, on $S$ is an abstract simplicial complex, introduced by Harvey $[\mathrm{H}]$, with vertex set $\mathcal{A}$, the set of isotopy classes of nontrivial simple closed curves, such that a set of $n+1$ vertices $\left\{a_{0}, a_{1}, a_{2}, \ldots, a_{n}\right\}$ forms an $n$-simplex if and only if $a_{0}, a_{1}, a_{2}, \ldots, a_{n}$ have pairwise disjoint representatives. The automorphism group of the complex of curves is isomorphic to the extended mapping class group modulo the 
center, except for the cases $(g, r) \in\{(0,2),(0,3),(0,4),(1,0),(1,1),(1,2)\}$. The reader is referred to $[\mathrm{Iv},[\mathrm{K}]$ and $[\mathrm{L}$ for proof of these results.

Let $\mathcal{B}$ denote the set of isotopy classes of nonseparating simple closed curves on $S$. The complex of nonseparating curves, $\mathcal{N}(S)$, is the subcomplex of $\mathcal{C}(S)$ with the vertex set $\mathcal{B}$ such that a set of $n+1$ vertices $\left\{b_{0}, b_{1}, b_{2}, \ldots, b_{n}\right\}$ forms an $n$-simplex if and only if it is an $n$-simplex in $\mathcal{C}(S)$. If $g \geq 2$, the automorphism group of $\mathcal{N}(S)$ is isomorphic to the extended mapping class group of $S$ modulo its center, by the results given in Ir3].

In $[\mathrm{Sc}$, Schmutz Schaller defined a graph $\mathcal{G}(S)$; the vertex set is again $\mathcal{B}$, the set of isotopy classes of nonseparating simple closed curves, and two vertices $a$ and $b$ are connected by an edge if and only if $i(a, b)=1$. He defines the graph $\mathcal{G}(S)$ for surfaces of genus zero as well, but we will not mention that case here. His main result is the following theorem; we state as much as we need in this paper. Notice that in the case $g=1$, since the vertices of $\mathcal{G}(S)$ can be viewed as vertices in $\mathcal{H} \mathcal{T}(S)$, the complex $\mathcal{G}(S)$ can be considered as a subcomplex of $\mathcal{H} \mathcal{T}(S)$ in a natural way. In fact, $\mathcal{G}(S)$ is the 1-skeleton of $\mathcal{H} \mathcal{T}(S)$.

Theorem 2. ( $\mathrm{Sc}]$ ) Let $S$ be a compact, connected, orientable surface of positive genus. Then Aut $\mathcal{G}(S)$ is isomorphic to the extended mapping class group $\operatorname{Mod}_{S}^{*}$ modulo the center.

In $\underline{\mathrm{Sc}}$, the case $(g, r)=(1,0)$ is not included, but clearly it follows from the case $(g, r)=(1,1)$.

The complex $X_{C}$. For a nonseparating simple closed curve $C$ on $S$, we define a simplicial complex (graph) $X_{C}$ as follows: the vertices of $X_{C}$ are isotopy classes of nonseparating simple closed curves which are dual to $C$ on $S$. A set $\{a, b\}$ of vertices forms an edge if and only if $a$ is dual to $b$.

We will need the following definiton in Lemma 3 An embedded arc $\epsilon$ on a surface $S$ with boundary is called properly embedded if $\partial \epsilon \subseteq \partial S$ and $\epsilon$ is transversal to $\partial S$. It is called nontrivial (or essential) if $\epsilon$ cannot be deformed into $\partial S$ in such a way that the endpoints of $\epsilon$ stay in $\partial S$ during the deformation.

Lemma 3. If $S$ is a connected orientable surface of positive genus and if $C$ is a nonseparating simple closed curve on $S$, then the complex $X_{C}$ is connected.

Proof. Let $d$ and $d^{\prime}$ be two distinct vertices in $X_{C}$. We will show that there is a path $d=d_{0} \rightarrow d_{1} \rightarrow \cdots \rightarrow d_{n+1}=d^{\prime}$ in $X_{C}$.

Let $D$ and $D^{\prime}$ be representatives of $d$ and $d^{\prime}$ respectively such that $D$ and $D^{\prime}$ have minimal intersection and that they are both dual to $C$. We may assume, moreover, that they intersect $C$ at different points. 
If $\left|D \cap D^{\prime}\right|=0$, then $d \rightarrow t_{c}(d) \rightarrow d^{\prime}$, where $t_{c}$ is the Dehn twist about $c$, is the path in $X_{C}$ that we want.

If $\left|D \cap D^{\prime}\right|=1$, then $d \rightarrow d^{\prime}$ is the required sequence.

Assume that $\left|D \cap D^{\prime}\right|=m>1$. Let $N$ be a regular neighborhood of $C$ such that the intersection of $D \cup D^{\prime}$ and $N$ is a pair of disjoint arcs. Let $R$ be the complement of the interior of $N$ in $S$ and let $\partial_{1}$ and $\partial_{2}$ be the boundary components of $N$. Let $\epsilon$ and $\tau$ denote the part of $D$ and $D^{\prime}$ on $R$ respectively, which are essential properly embedded arcs. We orient $\epsilon$ and $\tau$ so that they both start on $\partial_{1}$ and end on $\partial_{2}$. We define an arc in the following way: Start on the boundary component $\partial_{1}$ of $R$, on one side of the beginning point of $\tau$ and continue along $\tau$ without intersecting $\tau$, till the last intersection point of $\epsilon$ and $\tau$ along $\epsilon$. Then we would like to follow $\epsilon$, without intersecting $\epsilon \cup \tau$, until we reach $\partial_{2}$. So, if we are on the correct side of $\tau$ we do this; if not, we change our starting side from the beginning and follow the construction. This gives us an arc, say $\tau_{1}$. We see that $\tau_{1}$ is an essential properly embedded arc since it connects two boundary components $\partial_{1}$ and $\partial_{2}$, and $\left|\epsilon \cap \tau_{1}\right|<m$ since we eliminated at least one intersection with $\epsilon$. We also have $\left|\tau_{1} \cap \tau\right|=0$ since we never intersected $\tau$.

Now, using $\epsilon$ and $\tau_{1}$ in the place of $\epsilon$ and $\tau$ we define a new properly embedded arc $\tau_{2}$ connecting $\partial_{1}$ to $\partial_{2}$ such that $\left|\epsilon \cap \tau_{2}\right|<\left|\epsilon \cap \tau_{1}\right|,\left|\tau_{1} \cap \tau_{2}\right|=0$. By an inductive argument, we get a sequence

$$
\epsilon=\tau_{n+1} \rightarrow \tau_{n} \rightarrow \tau_{n-1} \rightarrow \cdots \rightarrow \tau_{1} \rightarrow \tau_{0}=\tau
$$

of essential properly embedded arcs on $R$ such that every consecutive pair is disjoint. So, $\epsilon=\tau_{n+1}$ and $\tau_{n}$ are two disjoint arcs on $R$. Note that $\epsilon=\tau_{n+1}$ is an arc of $D$ on $R$. We now connect the end points of $\tau_{n}$ with an arc in the interior of $N$ to get a nonseparating simple closed curve, $Q_{n}$, dual to both $C$ and $D$ on $S$. Then, we connect the end points of $\tau_{n-1}$ with an arc in the interior of $N$ so that we get a nonseparating simple closed curve, $Q_{n-1}$, which is dual to both $C$ and $\tau_{n}$ on $S$. Now by an inductive argument, we see that there is a sequence

$$
D=Q_{n+1} \rightarrow Q_{n} \rightarrow Q_{n-1} \rightarrow \cdots \rightarrow Q_{1} \rightarrow Q_{0}=Q
$$

consisting of nonseparating simple closed curves dual to $C$ on $S$ such that every consecutive pair is dual. So, $d$ is connected to $q$ by a path in $X_{C}$.

Since the parts of $D^{\prime}$ and $Q$ on $R$ are equal, we see that $q=t_{c}^{m}\left(d^{\prime}\right)$ for some $m \in \mathbb{Z}$. Then $q$ and $d^{\prime}$ can be connected by the path, $q=t_{c}^{m}\left(d^{\prime}\right) \rightarrow$ $t_{c}^{m-1}\left(d^{\prime}\right) \rightarrow \cdots \rightarrow t_{c}\left(d^{\prime}\right) \rightarrow d^{\prime}$ in $X_{C}$. Since $d$ and $q$ are connected by a path in $X_{C}$, we see that $d$ and $d^{\prime}$ are connected by a path in $X_{C}$. Hence, the complex $X_{C}$ is connected.

\section{Action of AUtomorphisms of $\mathcal{H} \mathcal{T}(S)$ ON NONSEPARAting CURVES}

We define an action of the automorphism group Aut $\mathcal{H} \mathcal{T}(S)$ of $\mathcal{H} \mathcal{T}(S)$ on the set of nonseparating simple closed curves as follows. Let $f: \mathcal{H T}(S) \rightarrow$ $\mathcal{H} \mathcal{T}(S)$ be an automorphism of the Hatcher-Thurston complex of the surface 
$S$. For an isotopy class $c$ of a nonseparating simple closed curve $C$, choose pairwise disjoint nonseparating simple closed curves $C_{2}, C_{3}, \ldots, C_{g}$ on $S$ such that $v=\left\langle c, c_{2}, \ldots, c_{g}\right\rangle$ is a cut system. Choose another curve $D$ on $S$ such that $C$ and $D$ are dual and $D$ does not intersect any of $C_{i}$. Then $w=\left\langle d, c_{2}, \ldots, c_{g}\right\rangle$ is also a cut system and the vertices $v$ and $w$ are connected by an edge in the complex $\mathcal{H} \mathcal{T}(S)$. Since $f$ is an automorphism, the vertices $f(v)$ and $f(w)$ are connected by an edge as well. Thus the difference $f(v)-$ $f(w)$ of the sets $f(v)$ and $f(w)$ contains only one curve. We define $\tilde{f}(c)$ to be this unique class.

Notice that if $g=1$ then the cut system $v$ contains only one element; $v=\langle c\rangle$. Thus $\tilde{f}(c)$ is the unique class in $f\langle c\rangle$, so that we have $\langle\tilde{f}(c)\rangle=f\langle c\rangle$.

Lemma 4. For a fixed set of curves $\left\{C_{2}, C_{3}, \ldots, C_{g}\right\}$, the definition of $\tilde{f}(c)$ is independent of the choice of the curve $D$.

Proof. Let $v_{1} \leftrightarrow v_{2} \leftrightarrow v_{3} \leftrightarrow v_{1}$ be a triangle in the complex $\mathcal{H} \mathcal{T}(S)$. Then we observe that $v_{1}-v_{2}=v_{1}-v_{3}$.

For a nonseparating simple closed curve $A$ such that $\left\langle a, c_{2}, \ldots, c_{g}\right\rangle$ is a cut system, let $\langle a\rangle$ denote the cut system $\left\langle a, c_{2}, \ldots, c_{g}\right\rangle$.

Let $D^{\prime}$ be a simple closed curve on $S$ such that it is dual to both $C$ and $D$, and disjoint from $C_{i}$ for $i \geq 2$. Then $\left\langle d^{\prime}\right\rangle=\left\langle d^{\prime}, c_{2}, \ldots, c_{g}\right\rangle$ is also a cut system and $\langle c\rangle \leftrightarrow\langle d\rangle \leftrightarrow\left\langle d^{\prime}\right\rangle \leftrightarrow\langle c\rangle$ is a triangle in $\mathcal{H} \mathcal{T}(S)$. Since $f$ is an automorphism, $f\langle c\rangle \leftrightarrow f\langle d\rangle \leftrightarrow f\left\langle d^{\prime}\right\rangle \leftrightarrow f\langle c\rangle$ is also a triangle in $\mathcal{H} \mathcal{T}(S)$. By the observation above we have $f\langle c\rangle-f\langle d\rangle=f\langle c\rangle-f\left\langle d^{\prime}\right\rangle$.

Suppose now that $D^{\prime}$ is an arbitrary simple closed curve on $S$ which is dual to $C$ and is disjoint from all $C_{i}$ for $i \geq 2$. Then $d$ and $d^{\prime}$ are two vertices of the complex $X_{C}$. Since this complex is connected by Lemma 3 , there is a sequence $d=d_{1}, d_{2}, \ldots, d_{n}=d^{\prime}$ of vertices in $X_{C}$ such that $d_{i}$ is connected to $d_{i+1}$ by an edge for all $i=1,2, \ldots, n-1$. By the previous paragraph, we have $f\langle c\rangle-f\left\langle d_{i}\right\rangle=f\langle c\rangle-f\left\langle d_{i+1}\right\rangle$. It follows that $f\langle c\rangle-f\langle d\rangle=f\langle c\rangle-f\left\langle d^{\prime}\right\rangle$.

This proves the lemma.

Lemma 5. For a nonseparating simple closed curve $C$, the definition of $\tilde{f}(c)$ is independent of all choices.

Proof. Suppose that $\left\{C_{2}, C_{3}, \ldots, C_{g}, D\right\}$ and $\left\{C_{2}^{\prime}, C_{3}^{\prime}, \ldots, C_{g}^{\prime}, D^{\prime}\right\}$ are two choices in the definition of $\tilde{f}(c)$. We must prove that both choices give rise to the same result. More precisely, if $v, w, v^{\prime}$ and $w^{\prime}$ denote the cut systems $\left\langle c, c_{2}, \ldots, c_{g}\right\rangle,\left\langle d, c_{2}, \ldots, c_{g}\right\rangle,\left\langle c, c_{2}^{\prime}, \ldots, c_{g}^{\prime}\right\rangle$ and $\left\langle d^{\prime}, c_{2}^{\prime}, \ldots, c_{g}^{\prime}\right\rangle$ respectively such that $v \leftrightarrow w$ and $v^{\prime} \leftrightarrow w^{\prime}$, then we must show that $f\left(v^{\prime}\right)-f\left(w^{\prime}\right)=$ $f(v)-f(w)$.

If $g=1$ then there are no $C_{i}$ and $C_{i}^{\prime}$ and the conclusion of the lemma follows from Lemma 4 . So we assume that $g \geq 2$.

Suppose first that $v^{\prime}$ is connected by an edge to $v$. Therefore there are elements $c_{i_{0}} \in v$ and $c_{j_{0}}^{\prime} \in v^{\prime}$ such that $C_{i_{0}}$ and $C_{j_{0}}^{\prime}$ intersect transversely at one point and $v-\left\{c_{i_{0}}\right\}=v^{\prime}-\left\{c_{j_{0}}^{\prime}\right\}$. After reindexing if necessary we can assume that $c_{i_{0}}=c_{2}$ and $c_{j_{0}}^{\prime}=c_{2}^{\prime}$, so that $c_{i}^{\prime}=c_{i}$ for $i \geq 3$. Let $E$ be 
a simple closed curve dual to $C$ and disjoint from all $C_{i}$ and $C_{2}^{\prime}$. Let $t=$ $\left\langle e, c_{2}, c_{3}, \ldots, c_{g}\right\rangle$ and $t^{\prime}=\left\langle e, c_{2}^{\prime}, c_{3}^{\prime}, c_{4}^{\prime}, \ldots, c_{g}^{\prime}\right\rangle=\left\langle e, c_{2}^{\prime}, c_{3}, c_{4}, \ldots, c_{g}\right\rangle$. Since $v \leftrightarrow v^{\prime} \leftrightarrow t^{\prime} \leftrightarrow t \leftrightarrow v$ form a rectangle in $\mathcal{H} \mathcal{T}(S)$ and $f$ is an automorphism, $f(v) \leftrightarrow f\left(v^{\prime}\right) \leftrightarrow f\left(t^{\prime}\right) \leftrightarrow f(t) \leftrightarrow f(v)$ is a rectangle in $\mathcal{H} \mathcal{T}(S)$. Then it is easy to see that $f(v)-f(t)=f\left(v^{\prime}\right)-f\left(t^{\prime}\right)$. By using Lemma 4 we obtain $f(v)-f(t)=f(v)-f(w)$ and $f\left(v^{\prime}\right)-f\left(t^{\prime}\right)=f\left(v^{\prime}\right)-f\left(w^{\prime}\right)$. Therefore, we get the desired result $f(v)-f(w)=f\left(v^{\prime}\right)-f\left(w^{\prime}\right)$.

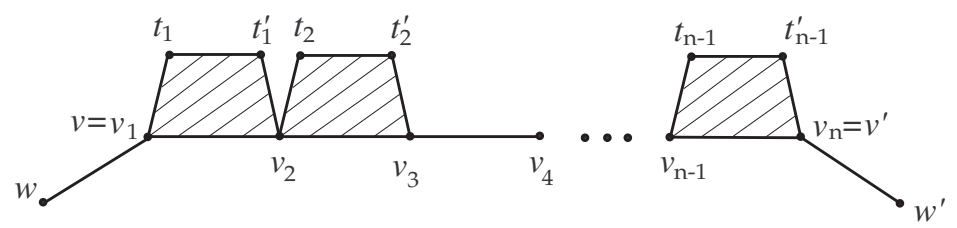

Figure 2. A path in $\mathcal{H} \mathcal{T}(S)$

Let us now consider the general case. Let $R$ denote the surface obtained by cutting $S$ along the curve $C$. Thus $R$ is a surface of positive genus. Since all $C_{i}$ and $C_{i}^{\prime}, i \geq 2$, are disjoint from $C$, we can consider them as curves on $R$. Now $V=\left\langle c_{2}, c_{3}, \ldots, c_{g}\right\rangle$ and $V^{\prime}=\left\langle c_{2}^{\prime}, c_{3}^{\prime}, \ldots, c_{g}^{\prime}\right\rangle$ are two cut systems on $R$. Since the Hatcher-Thurston complex $\mathcal{H} \mathcal{T}(R)$ is connected Theorem 1 there is a sequence $V=V_{1}, V_{2}, V_{3}, \ldots, V_{n}=V^{\prime}$ of cut systems on $R$ such that $V_{i}$ is connected by an edge to $V_{i+1}$. If we denote by $v_{i}$ the cut system on $S$ obtained from $V_{i}$ by adding $c$, we get a path $v=v_{1}, v_{2}, \ldots, v_{n}=v^{\prime}$ in $\mathcal{H} \mathcal{T}(S)$. For each $i=1,2, \ldots, g-1$, choose vertices $t_{i}$ and $t_{i}^{\prime}$ as in the previous paragraph such that $v_{i} \leftrightarrow v_{i+1} \leftrightarrow t_{i}^{\prime} \leftrightarrow t_{i} \leftrightarrow v_{i}$ is a rectangle. We showed above that $f\left(v_{i}\right)-f\left(t_{i}\right)=f\left(v_{i+1}\right)-f\left(t_{i}^{\prime}\right)$. By Lemma 4 we also have $f\left(v_{i+1}\right)-f\left(t_{i}^{\prime}\right)=f\left(v_{i+1}\right)-f\left(t_{i+1}\right)$. It follows that $f\left(v_{1}\right)-f\left(t_{1}\right)=$ $f\left(v_{n}\right)-f\left(t_{n-1}^{\prime}\right)$. Now the conclusion $f(v)-f(w)=f\left(v^{\prime}\right)-f\left(w^{\prime}\right)$ follows from Lemma 4 .

This completes the proof of the lemma.

Lemma 6. Let $c, d$ be the isotopy classes of two nonseparating simple closed curves $C$ and $D$ such that $i(c, d)=1$. Then $i(\tilde{f}(c), \tilde{f}(d))=1$.

Proof. It is easy to see that we can find nonseparating simple closed curves $C_{2}, C_{3}, \ldots, C_{g}$ on $S$ such that $v=\left\langle c, c_{2}, \ldots, c_{g}\right\rangle$ and $w=\left\langle d, c_{2}, \ldots, c_{g}\right\rangle$ are two vertices in $\mathcal{H} \mathcal{T}(S)$. Since the geometric intersection of $c$ and $d$ is 1 , we see that $v$ and $w$ are connected by an edge in $\mathcal{H} \mathcal{T}(S)$. Since $f$ is an automorphism $f(v)$ and $f(w)$ are also connected by an edge in $\mathcal{H} \mathcal{T}(S)$. From the definition of $\tilde{f}$, we have $\{\tilde{f}(c)\}=f(v)-f(w)$ and $\{\tilde{f}(d)\}=$ $f(w)-f(v)$. Since $f(v)$ and $f(w)$ are connected by an edge, we conclude that $i(\tilde{f}(c), \tilde{f}(d))=1$. 
Lemma 7. If $f$ and $h$ are two automorphisms of $\mathcal{H T}(S)$ and if $c$ is the isotopy class of a nonseparating simple closed curve $C$, then $\widetilde{f h}(c)=\tilde{f}(\tilde{h}(c))$.

Proof. Let us choose vertices $c_{2}, c_{3}, \ldots, c_{g}$ and $d$ in $\mathcal{G}(S)$ such that $v=$ $\left\langle c, c_{2}, \ldots, c_{g}\right\rangle$ and $w=\left\langle d, c_{2}, \ldots, c_{g}\right\rangle$ are distinct vertices in the complex $\mathcal{H} \mathcal{T}(S)$ which are connected by an edge; $v \leftrightarrow w$. Then $\{\tilde{h}(c)\}=h(v)-h(w)$ and $\{\widetilde{f h}(c)\}=f h(v)-f h(w)$. Since $h(v) \leftrightarrow h(w)$, we can use these vertices to define $\tilde{f}(\tilde{h}(c))$ :

$$
\begin{aligned}
\{\tilde{f}(\tilde{h}(c))\} & =f(h(v))-f(h(w)) \\
& =(f h)(v)-(f h)(w) \\
& =\{(\widetilde{f h})(c)\} .
\end{aligned}
$$

Proposition 8. The mapping $\tilde{f}$ is an automorphism of the graph $\mathcal{G}(S)$.

Proof. For a vertex $c$ in $\mathcal{G}(S), \tilde{f}(c)$ is well-defined. Therefore we have a well-defined map $\tilde{f}: \mathcal{G}(S) \rightarrow \mathcal{G}(S)$. If two vertices $c, d$ are connected by an edge in $\mathcal{G}(S)$, then $i(c, d)=1$. By Lemma $[i(\tilde{f}(c), \tilde{f}(d))=1$. Therefore, $\tilde{f}$ is simplicial.

Let $h \in \operatorname{Aut} \mathcal{H} \mathcal{T}(S)$ be the inverse of $f$. Then $\tilde{f} \tilde{h}$ and $\tilde{h} \tilde{f}$ are both the identity automorphisms, because it can be shown that if $I \in \operatorname{Aut} \mathcal{H} \mathcal{T}(S)$ denote the identity, then $\tilde{I}(c)=c$ for all nonseparating simple closed curve $C$. We conclude that $\tilde{f}: \mathcal{G}(S) \rightarrow \mathcal{G}(S)$ is a bijection.

In the following proposition we will prove that $\tilde{f}$ preserves geometric intersection zero, and hence also is an automorphism of $\mathcal{N}(S)$ for closed surfaces.

Proposition 9. If $S$ is a closed surface of genus at least two, then the mapping $\tilde{f}: \mathcal{N}(S) \rightarrow \mathcal{N}(S)$ is an automorphism.

Proof. By the previous proposition, $\tilde{f}$ is a bijection. So, it is enough to show that $\tilde{f}$ is a simplicial map on $\mathcal{N}(S)$. Let $a, b$ be two distinct vertices of $\mathcal{N}(S)$, which have disjoint representatives $A$ and $B$ on $S$ respectively. We will consider the following two cases:

Case i: If $S_{A \cup B}$ is connected, then $\{a, b\}$ can be completed to a vertex $v$ in $\mathcal{H} \mathcal{T}(S)$. Then, since $f(v)$ is a vertex in $\mathcal{H} \mathcal{T}(S)$ and $\tilde{f}(a), \tilde{f}(b) \in f(v)$, we see that $\tilde{f}(a)$ and $\tilde{f}(b)$ have disjoint representatives on $S$.

Case ii: If $S_{A \cup B}$ is not connected, then we complete $A$ and $B$ to a curve configuration as shown in Figure 3 , by taking a maximal chain $\left\{C_{1}, \ldots, C_{2 g+1}\right\}$ with $i\left(c_{i}, c_{i+1}\right)=1, i\left(c_{i}, c_{j}\right)=0$ for $|i-j|>1, c_{i} \in \mathcal{N}(S)$ as shown in the figure for $g=4$ case (similar chains can be chosen in the other cases). Notice that $S_{C_{i} \cup C_{j}}$ is connected for any $i, j$. So, if $i\left(c_{i}, c_{j}\right)=0$ then $i\left(\tilde{f}\left(c_{i}\right), \tilde{f}\left(c_{j}\right)\right)=$ 0 by the first case. If $i\left(c_{i}, c_{j}\right)=1$, then $i\left(\tilde{f}\left(c_{i}\right), \tilde{f}\left(c_{j}\right)\right)=1$ by Lemma 6 Hence $\left\{\tilde{f}\left(c_{1}\right), \ldots, \tilde{f}\left(c_{2 g+1}\right)\right\}$ is a maximal chain on $S$. 


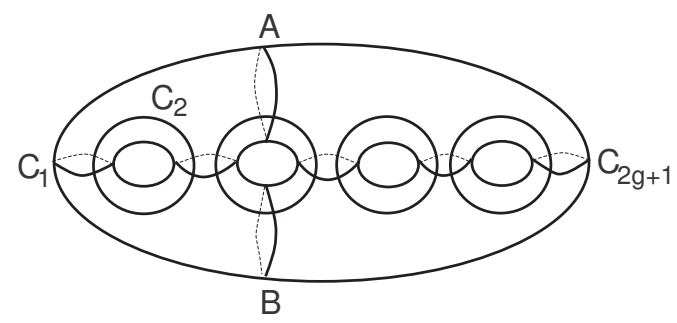

Figure 3. A, B and a chain

We also have that $S_{A \cup C_{i}}$ is connected for any $i$. So, if $i\left(a, c_{i}\right)=0$ then $i\left(\tilde{f}(a), \tilde{f}\left(c_{i}\right)\right)=0$ by the first case. If $i\left(a, c_{i}\right)=1$, then $i\left(\tilde{f}(a), \tilde{f}\left(c_{i}\right)\right)=1$ by Lemma 6. Similarly since $S_{B \cup C_{i}}$ is connected for any $i$, if $i\left(b, c_{i}\right)=0$ then $i\left(\tilde{f}(b), \tilde{f}\left(c_{i}\right)\right)=0$ by the first case. If $i\left(b, c_{i}\right)=1$, then $i\left(\tilde{f}(b), \tilde{f}\left(c_{i}\right)\right)=1$ by Lemma 6 .

Note that $i\left(a, c_{2 k}\right)=i\left(b, c_{2 k}\right)=1$ for some integer $k \in\{2,3, \ldots, g-1\}$ and the intersection numbers of $a$ and $b$ with any other $c_{i}$ is 0 . Therefore, $i\left(\tilde{f}(a), \tilde{f}\left(c_{2 k}\right)\right)=i\left(\tilde{f}(b), \tilde{f}\left(c_{2 k}\right)\right)=1$ and the intersection numbers of $\tilde{f}(a)$ and $\tilde{f}(b)$ with any other $\tilde{f}\left(c_{i}\right)$ is 0 .

Let $C_{i}^{\prime} \in \tilde{f}\left(c_{i}\right), A^{\prime} \in \tilde{f}(a)$ and $B^{\prime} \in \tilde{f}(b)$ such that all the curves $C_{i}^{\prime}, A^{\prime}$ and $B^{\prime}$ intersect minimally with each other for each $i$. Since $A$ and $B$ are dual to $C_{2 k}, A^{\prime}$ and $B^{\prime}$ are dual to $C_{2 k}^{\prime}$ by Lemma 6. Since curves $A^{\prime}$ and $B^{\prime}$ are disjoint from the chains $C_{1}^{\prime} \cup C_{2}^{\prime} \cup \cdots \cup C_{2 k-1}^{\prime}$ and $C_{2 k+1}^{\prime} \cup C_{2 k+2}^{\prime} \cup \cdots \cup C_{2 g+1}^{\prime}$ and since the complement of these two chains is the union of two annuli, the (distinct) curves $A^{\prime}$ and $B^{\prime}$ must be disjoint. Because up to isotopy there are only two simple closed curves on the disjoint union of two annuli and they are disjoint. Therefore, $i(\tilde{f}(a), \tilde{f}(b))=0$. This shows that $\tilde{f}$ is a simplicial map on $\mathcal{N}(S)$. Since $\tilde{f}$ is 1-1 and onto, it is an automorphism of $\mathcal{N}(S)$.

Remark: If $S$ is a closed surface of genus at least two, by using Proposition 9 and the results in [Ir3], we see that $f$ is induced by a homeomorphism of $S$.

\section{Automorphisms of $\mathcal{H} \mathcal{T}(S)$ and mapping class group}

In this final section, we state and prove the main result. We then give a corollary to the main theorem and comment on other possible but similar proofs of the main theorem.

Theorem 10. Let $S$ be a compact, connected, orientable surface of genus at least one. Then the mapping $\varphi:$ Aut $\mathcal{H} \mathcal{T}(S) \rightarrow \operatorname{Aut} \mathcal{G}(S)$ given by $f \mapsto \tilde{f}$ is an isomorphism. 
Proof. By the results of the previous section, $\varphi(f)=\tilde{f}$ is a well-defined automorphism of Aut $\mathcal{G}(S)$, and Lemma 7 shows that $\varphi$ is a group homomorphism.

For an element $f \in \operatorname{Aut} \mathcal{H} \mathcal{T}(S)$ if $\tilde{f}$ is the identity automorphism of $\mathcal{G}(S)$, then it follows from $f\left(\left\langle c_{1}, c_{2}, \ldots, c_{g}\right\rangle\right)=\left\langle\tilde{f}\left(c_{1}\right), \tilde{f}\left(c_{2}\right), \ldots, \tilde{f}\left(c_{g}\right)\right\rangle$ that $f$ acts trivially on $\mathcal{H} \mathcal{T}(S)$. Hence, $\varphi$ is one-to-one.

If $h$ is an automorphism of $\mathcal{G}(S)$, then $h$ is induced by a homeomorphism $F$ of the surface $S$ by the results given in $\underline{\underline{S c}}$. Now $F$ induces an automorphism $f$ of $\mathcal{H} \mathcal{T}(S)$ and $\tilde{f}=h$. Hence, $\varphi$ is an isomorphism. This completes the proof of the theorem.

Corollary 11. Let $S$ be a compact, connected, orientable surface of genus $g \geq 1$ with $r \geq 0$ boundary components. If $(g, r) \neq(1,0),(1,1),(1,2),(2,0)$, then we have Aut $\mathcal{H} \mathcal{T}(S) \cong \operatorname{Mod}_{S}^{*}$. If $(g, r)$ is one of $(1,0),(1,1),(1,2),(2,0)$, then we have Aut $\mathcal{H} \mathcal{T}(S) \cong \operatorname{Mod}_{S}^{*} / \mathbb{Z}_{2}$. That is, in all cases Aut $\mathcal{H} \mathcal{T}(S) \cong$ $\operatorname{Mod}_{S}^{*} / \mathcal{C}\left(\operatorname{Mod}_{S}^{*}\right)$.

Proof. The proof follows from Theorem [10 and Theorem 2

This gives us another evidence to the following conjectural statement; the automorphism group of any natural complex on curves is isomorphic to the extended mapping class group in generic cases. By using our results given in this paper, and the main results of [Ir1, Ir2, Ir3, Iv], $\mathrm{Sc}$, IM], we see that for most of the compact, connected, orientable surfaces, we have Aut $\mathcal{H} \mathcal{T}(S) \cong \operatorname{Aut} \mathcal{N}(S) \cong \operatorname{Aut} \mathcal{C}(S) \cong \operatorname{Aut} \mathcal{G}(S) \cong \operatorname{Aut} \mathcal{P}(S)$ where $\mathcal{P}(S)$ is the pants complex.

If $S$ is a closed surface of genus at least two and $f$ is an automorphism of $\mathcal{H} \mathcal{T}(S)$, by using the techniques given in this paper, in particular Lemma 6. we can see that $f$ induces an automorphism $\tilde{f}_{*}$ on $\mathcal{C}(S)$ by extending $\tilde{f}$ over the nontrivial separating curves on $S$ by using chains on two subsurfaces that the separating curves separate. We do the following: Let $C$ be a nontrivial separating curve on $S$. Since $g \geq 2, C$ separates $S$ into two subsurfaces $S_{1}, S_{2}$, and both of $S_{1}, S_{2}$ have genus at least one. We take a chain on $S_{1},\left\{a_{1}, a_{2}, \ldots, a_{m}\right\}$ with $i\left(a_{i}, a_{i+1}\right)=1, i\left(a_{i}, a_{j}\right)=0$ for $|i-j|>1, a_{i} \in \mathcal{N}(S)$, such that $S_{1} \cup\{c\}$ is a regular neighborhood of $A_{1} \cup A_{2} \cup \cdots \cup A_{n}$ where $A_{i} \in a_{i}$ and $A_{i}$ 's intersect minimally. Since $\tilde{f}$ preserves disjointness and intersection one property, we can see that the chain $\left\{a_{1}, a_{2}, \ldots, a_{m}\right\}$ is mapped by $\tilde{f}$ into a similar chain, $\left\{\tilde{f}\left(a_{1}\right), \ldots, \tilde{f}\left(a_{m}\right)\right\}$ with $i\left(\tilde{f}\left(a_{i}\right), \tilde{f}\left(a_{i+1}\right)\right)=1, i\left(\tilde{f}\left(a_{i}\right), \tilde{f}\left(a_{j}\right)\right)=0$ for $|i-j|>1$. Let $A_{i}^{\prime} \in \tilde{f}\left(a_{i}\right)$ such that any two elements in $\left\{A_{1}^{\prime}, \ldots, A_{m}^{\prime}\right\}$ have minimal intersection with each other. Let $M$ be a regular neighborhood of $A_{1}^{\prime} \cup A_{2}^{\prime} \cup \ldots \cup A_{m}^{\prime}$. Then it is easy to see that $M$ is homeomorphic to $R_{1} \cup c$. Let $C^{\prime}$ be the boundary of $M$. We define $\tilde{f}_{*}(c)=\left[C^{\prime}\right]$ (See [Ir3 for well definedness). This extends $\tilde{f}$ to a simplicial map $\tilde{f}_{*}$ on $\mathcal{C}(S)$. It can be shown that $\tilde{f}_{*}$ is an automorphism on $\mathcal{C}(S)$. 


\section{Acknowledgments}

We would like to thank Joan Birman, Nikolai Ivanov, John McCarthy and Dan Margalit for their interest in this work and for valuable comments about this paper.

\section{REFERENCES}

[BM] T.E. Brendle, D. Margalit, Commensurations of the Johnson kernel. Geometry and Topology, 8 (2004), 1361-1384.

[CC] R. Charney, J. Crisp, Automorphism groups of some affine and finite type Artin groups. Universite de Bourgogne, Institut de Mathematiques Preprint No 385.

[FI] B. Farb, N.V. Ivanov, The Torelli geometry and its applications. arXiv:math.GT/0311123

[H] W.J. Harvey, Geometric structures of surface mapping class groups, Homological Group Theory (C.T. Wall, ed), London Math. Soc. Lecture Notes, No.36, Cambridge Univ. Press, London, 1979, 255-269.

[Ha] J. Harer The second homology group of the mapping class group of an orientable surface. Invent. Math. 72 (1983), no. 2, 221-239.

[HT] A. Hatcher, W. Thurston A presentation for the mapping class group of a closed orientable surface Topology 19, 221-237 (1980).

[Ir1] E. Irmak, Superinjective simplicial maps of complexes of curves and injective homomorphisms of subgroups of mapping class groups, Topology 43 (2004), No.3, $513-541$.

[Ir2] E. Irmak, Superinjective simplicial maps of complexes of curves and injective homomorphisms of subgroups of mapping class groups II, Topology and Its Applications to appear, http://front.math.ucdavis.edu/math.GT/0311407

[Ir3] E. Irmak, Complexes of nonseparating curves and mapping class groups, submitted, available at http://front.math.ucdavis.edu/math.GT/0407285

[Iv] N.V. Ivanov, Automorphisms of complexes of curves and of Teichmuller spaces, International Mathematics Research Notices, No.14 1997, 651-666.

[K] M. Korkmaz, Automorphisms of complexes of curves on punctured spheres and on punctured tori. Topology and its Applications, 95 (2) (1999), 85-111.

[L] F. Luo, Automorphisms of complexes of curves, Topology 39 (2000) 283-298.

[M] D. Margalit, Automorphisms of the pants complex, Duke Mathematical Journal 121, 457-479, 2004.

[MW] H. Masur, M. Wolf, The Weil-Petersson isometry group. Geom. Dedicata 93 (2002), 177-190.

[Sc] P. Schmutz Schaller, Mapping class groups of hyperbolic surfaces and automorphism groups of graphs, Composito Mathematica 122 (2000), 243-260.

[W1] B. Wajnryb, A simple presentation for the mapping class group of an orientable surface, Israel J. Math. 45 (1983), 157-174.

[W2] B. Wajnryb, An elementary approach to the mapping class group of a surface, Geom. Topol. 3 (1999), 405-466.

Department of Mathematics, University of Michigan, Ann Arbor, Mi 48109, USA

E-mail address: eirmak@umich.edu

Department of Mathematics, Middle East Technical University, 06531 Ankara, TURKEY

E-mail address: korkmaz@arf.math.metu.edu.tr 